\title{
ESTIMACIÓN DE COSTOS DE INVERSIÓN PARA UN ESTUDIO DE CASO: DREN TOPO Y DREN CON TUBERÍA EN LA REGIÓN CENTRAL SANTAFESINA
}

\author{
Camussi, G. ${ }^{1} ;$ Maina, M. ${ }^{2}$; Travadelo, M. $^{2}$ \& Marano, R. ${ }^{1}$
}

\begin{abstract}
RESUMEN
El período 1970- 2018 se caracteriza, en gran parte de la región pampeana argentina, por la ocurrencia de eventos con predominio de excesos hídricos que pueden mitigarse con la aplicación de técnicas de manejo integradas. El objetivo del trabajo fue estimar y comparar, aplicando la metodología del estudio de casos, los costos de las inversiones necesarias para implementar dos técnicas de manejo de excesos hídricos: dren topo y dren tubo. Los resultados obtenidos se expresaron en pesos (08/2018), resultando los montos de la inversión de 9.268 \$ ha-1 y 48.464 \$ ha-1, y de VAN de $\$ 106.319$ y $\$ 433.348$ para el dren topo y tubo respectivamente. Resulta necesario relevar información correspondiente al impacto de ambas técnicas a fin de poder evaluar estos proyectos con respecto a otras alternativas viables desde el punto de vista técnico y económico.
\end{abstract}

Key words: cuencas hidrográficas, excesos hídricos, tambos.

\begin{abstract}
Stimation of investment costs for a case study: the storm drain and pipe drain in the santafesina central regione.

The period 1970- 2018 is described, in a part of the Pampas region of Argentina, by the occurrence of extreme events with a predominance of water excesses. These problems can be mitigated through the application of integrated management techniques. The aim of this work was to estimate, applying the methodology of the case study, the costs of the investments necessary to implement
\end{abstract}

1.- Diagnóstico y Tecnología de Aguas, IDEA. Facultad de Ciencias Agrarias, Universidad Nacional del Litoral. Kreder 2805, (3080) Esperanza, provincia de Santa Fe. Email: mmaina@fca.unl.edu.ar 2.- Grupo de Economía Agraria. Facultad de Ciencias Agrarias, Universidad Nacional del Litoral. Manuscrito recibido el 19 de marzo de 2019 y aceptado para su publicación el 23 de junio de 2019.

Camussi, G.; Maina, M.; Travadelo, M \& Marano, R. Estimación de costos de inversión para un estudio de caso: dren topo y dren con tubería en la Región Central Santafesina. FAVE - Ciencias Agrarias 18 (1): 35-47. CC BY-NC-SA 4.0 (c) (i) (2) 


\section{G. Camussi et al.}

water management techniques: the storm drain and pipe drain. The results obtained was expressed in August 2.018 currency. The investment value for the mole drainage was $\$ /$ ha 9.268 and for the drain with pipe \$/ha 48.464 . The NPV was of \$ 106.319 and of \$ 433.348 for the alternative mole drainage and drain with pipe respectively. It is necessary to collect information corresponding to the impact of both techniques to be able to evaluate these projects with respect to other viable alternatives from the technical and economic point of view.

Key words: watersheds, water excesses, dairy.

\section{INTRODUCCIÓN}

El período 1970- 2018 se caracteriza, en gran parte de la región pampeana argentina, por la ocurrencia de eventos extremos con predominio de excesos hídricos, aunque también importantes sequías. Lovino (12) analizó, para igual período, lluvias acumuladas en 5 días y encontró un incremento de su magnitud. Los excesos hídricos generan encharcamiento, anegamientos y/o inundación de suelos productivos, así como también el incremento de los niveles freáticos, que incluso pueden alcanzan la superficie del suelo. Este tipo de amenazas naturales son las de mayor impacto para el normal desarrollo de las actividades agroproductivas (13). Fitzgerald et al. (8) evaluaron la interacción suelo-clima en sistemas de producción lechera, demostrando que suelos bien drenados posibilitan el sostenimiento de mayor carga animal.

En Santa Fe predominan sistemas hidrológicos no típicos, característicos de cuencas hidrográficas de llanura, ubicadas en regiones de climas húmedos y templados. Además del relieve plano, los suelos de esta Región presentan baja capacidad de infiltración y cambios en su uso, con una hidro-estructura (canales, cunetas, puentes, alcantarillas, caminos y rutas) que no está adaptada a la ocurrencia de lluvias de gran magnitud (7) (10).
Al respecto, según la encuesta sectorial lechera del INTA (11) en el período 2016 y 2017 se registraron lluvias abundantes y anegamientos en la región que afectaron negativamente el $60 \%$ de los tambos analizados. En términos productivos, se estimó una pérdida del 43\% en la superficie de las pasturas y la producción de leche diaria disminuyó un 46\% pasando de 2.258 l/d a 1.323 l/d. luego de 5 días posteriores al evento.

Estos problemas pueden mitigarse a través de la aplicación de técnicas de manejo integradas, es decir, técnicas vinculadas entre sí dentro del predio y a obras estructurales extra prediales (17)

En este sentido se pueden mencionar tres grandes técnicas (14):

a) drenaje, que tienen la finalidad de captar parte del escurrimiento superficial en suelos anegados (canales excavados) y/o de capas freáticas que provocan encharcamiento (drenes subsuperficiales), conduciendo estos excedentes hacia vías de escurrimiento o represas, tanto naturales como construidas ad hoc (20). La vinculación de estas técnicas prediales con la red de drenaje de la cuenca hidrográfica no es solo una cuestión de diseño, sino que debe cumplir con la legislación vigente (Ley de Aguas de Santa Fe). A modo de ejemplo, en su artículo 91 menciona "Se entenderá por obra hidráulica, a los efectos de esta ley, 
a toda construcción que implique el uso y control de los recursos hídricos y tenga por objeto la captación de aguas superficiales y subterráneas, medición, almacenamiento, regulación, derivación, conducción, obras de paso, alumbramiento, conservación, utilización o descontaminación del agua o defensa contra sus efectos nocivos”. Es decir que si no respeta la legislación pueden ser categorizadas como obras clandestinas.

b) reservorios, permitiendo maximizar la acumulación de agua en el suelo y mantenerlo próximo o cercano a capacidad de campo, así como almacenar el agua de escorrentía superficial o subsuperficial en cubetas naturales (hoyas de deflación) o construidos específicamente con diversos fines. Estos reservorios son intraprediales y permiten tanto retardar el escurrimiento superficial originado por lluvias extraordinarias como ser aprovechado a posteriori en ciclos con balance hídrico negativo (14).

c) riego suplementario, que en la región pampeana se utiliza para suplir posible déficit hídricos que se presentan en períodos cortos (una a dos semanas) o más prolongados (meses) (2) (15).

Respecto de las posibles técnicas a aplicar para este trabajo, se seleccionó drenaje subsuperficial debido a que los excesos hídricos presentan cada vez mayor recurrencia (12) con importante afectación de las pasturas. El drenaje subsuperficial puede ser: i) somero o poco profundo $(0,7$ a $0,8 \mathrm{~m}$ de profundidad), práctica conocida como dren topo (DT); ii) drenes tubo (DTu), instalados a mayor profundidad (hasta $2 \mathrm{~m}$ ) para control de la capa freática (18). DT tiene la particularidad de aprovechar horizontes con elevada proporción de arcilla para formar galerías subterráneas construidas con un implemento especialmente diseñado, a una profundidad variable entre 0,5 y $1 \mathrm{~m}$. El espaciamiento también es variable entre 4 a 8 m dependiendo de la profundidad y la conductividad hidráulica del estrato a drenar (15) (2).

Los extremos de estos drenes pueden ser abiertos o cerrados. En el primer caso se requiere de un colector de drenaje (principal o secundario) excavado por debajo de la profundidad de salida de los drenes (entre 0,7 y $0,9 \mathrm{~m}$ ), en tanto los últimos 2 a $3 \mathrm{~m}$ de cada dren deben contar con una conexión rígida (PVC o similar) para impedir que la galería colapse por erosión (Figura 1). Dado que este canal colector se ubica dentro del lote, presenta las siguientes desventajas: i) resta área productiva, ii) dificulta la conectividad entre lotes adyacentes, ii) necesita mantenimiento y iv) el gradiente de pendiente es insuficiente para conectar la zanja a la red de drenaje regional.

También debe considerarse para el diseño que, dada su ubicación en la parte más baja del lote, captará el excedente superficial reduciendo así la probabilidad de anegamiento, pero aumentando los costos de excavación.

En cambio, los drenes con final cerrado no tienen conexión y por lo general el flujo queda saturando el perfil del suelo generando un área de sacrificio no productiva. La efectividad y la vida útil de los drenes puede ser menor que en el caso anterior. Una opción es cruzar los extremos con drenes topo en sentido perpendicular y a la misma profundidad, pero espaciados a 1 o $2 \mathrm{~m}$ para conectar ambos drenes y conducirlos a un área de sacrificio que tiene vegetación espontánea (Figura 2).

1.- Ley de Aguas $N^{\circ} 13.740$ de la Provincia de Santa Fe acceso digital en julio de 2019 https://www.santafe. gov.ar/boletinoficial/recursos/boletines/16-02-2018ley13740-2018.html 
G. Camussi et al.

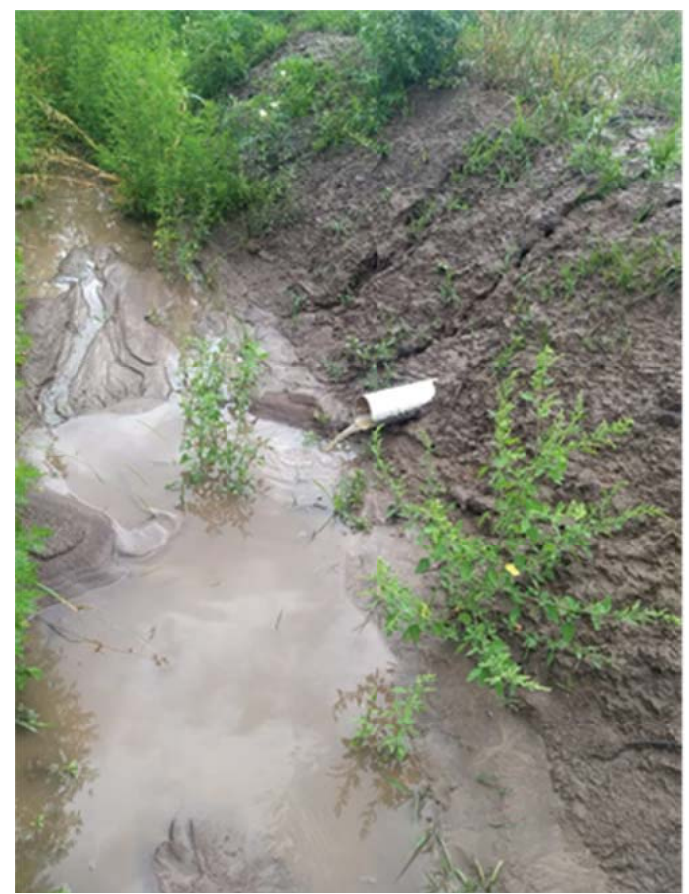

Figura 1. Drenes topo con final entubado conectado a una zanja de drenaje.

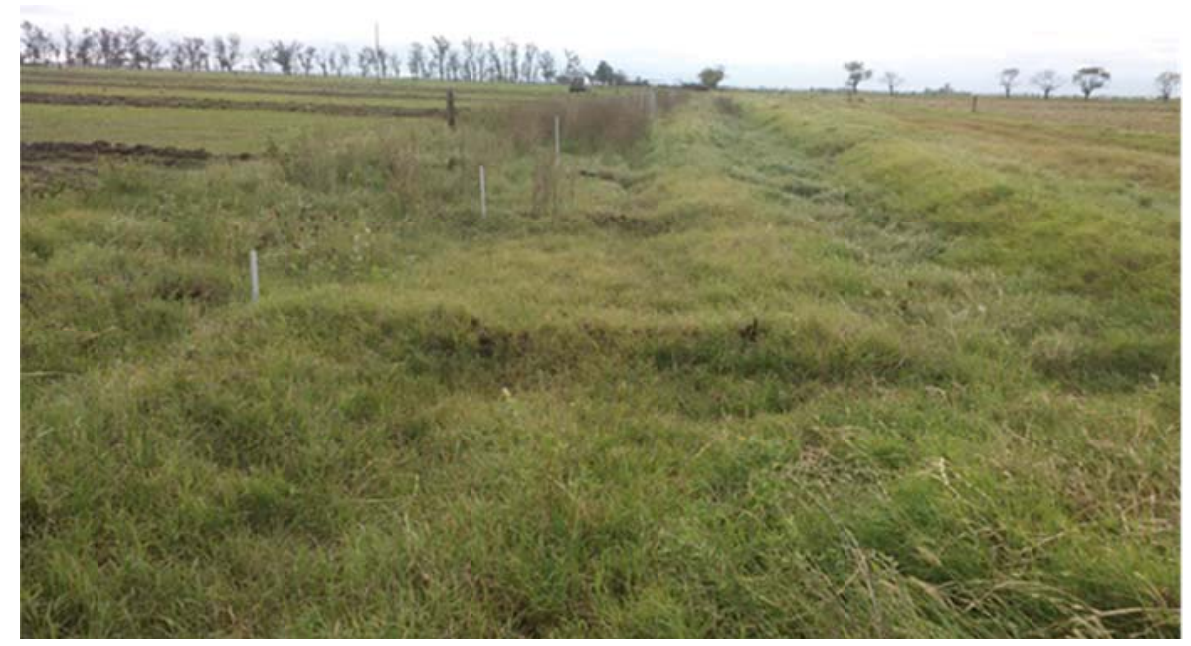

Figura 2. Drenes topo con final cerrado y área de sacrificio. 
Los drenes topo tienen una vida útil de aproximadamente tres años en la región considerada, luego de los cuales se debe volver a realizar la práctica con los implementos necesarios. Durante este período no se realizan tareas de mantenimiento.

El sistema de drenaje DTu se utiliza para control de la capa freática y consiste en la instalación de tubos corrugados de PVC o polietileno (PE) con pequeñas ranuras que permiten el ingreso del agua adyacente que satura los poros. Se instalan a profundidades variables (1 hasta 2,5 m) en función de la conductividad hidráulica de los estratos de suelo, profundidad de enraizamiento de las especies implantadas, presencia de capas duras o de roca, salinidad de la capa freática y maquinaria utilizada. En todos los casos son profundidades mayores que DT. La principal ventaja de este sistema es su mayor vida útil, que en promedio es de 15 a 20 años pero con costos de inversión mayores que los drenes topo (18).

Esta técnica es ampliamente utilizada en países como Holanda, España, Francia, Canadá y Estados Unidos pero en Argentina, debido a los altos costos de implementación, su instalación está restringida a las zonas bajo riego y solo en cultivos con alta rentabilidad (vid para vinos finos y en algunas especies frutales en Mendoza y San juan). En Jujuy se utiliza DTu con buenos resultados en la producción de caña de azúcar y en consecuencia mejorando la rentabilidad del negocio (21). La desventaja que se presenta es la falta de insumos específicos importados y maquinaria apropiada en Argentina para realizar las obras correspondientes.

En Irlanda se combina el uso de drenes topo y tubería de drenaje, con el primero ubicado en los horizontes superficiales del suelo, en tanto los tubos se instalan por debajo y en dirección perpendicular. De este modo DT aumenta la infiltración luego de un evento de lluvia y DTu controla el nivel freático (22).

Si bien se han instalado recientemente DT en la región central de Santa Fe (3), no se dispone de información actualizada respecto a los valores de las inversiones necesarias para llevarlas a cabo. Por lo tanto, para un caso de estudio ubicado en el departamento Las Colonias - provincia de Santa Fe-, se realizó una estimación de los costos de inversión y operación para implementar drenaje subsuperficial con DT y DTu a fin de identificar y proponer estrategias de intervención que mejoren el resultado productivo y económico de las empresas agropecuarias.

\section{MATERIALES Y MÉTODOS}

Se consideró la metodología correspondiente a Estudio de Casos propuesta por Yin (23) a fin de definir el caso de estudio. El citado autor plantea el "Estudio de Casos" como una investigación empírica que analiza un fenómeno contemporáneo dentro de su contexto real, en la que los límites entre el fenómeno y el contexto no son claramente visibles y sobre los cuales el investigador no tiene control.

La unidad de análisis seleccionada como caso de estudio corresponde a un lote de 8 ha destinado a la producción de forraje y semilla, ubicado en el departamento Las Colonias, provincia de Santa Fe.

Las etapas necesarias que fueron consideradas para la estimación de los costos de DT y DTu son: 


\section{G. Camussi et al.}

1.- Estudios planialtimétricos.

2.- Estudios de campo y laboratorio: conductividad hidráulica saturada, granulometría, profundidad y espesor del horizonte Bt (B textural), salinidad del suelo, profundidad de la freática y determinación del hidroapoyo o capa impermeable.

3.- Diseño red de la red drenaje (trabajo de gabinete) Determinación de insumos necesarios.

4.- Realización de las obras.

Con el objeto de estimar los costos de las inversiones necesarias para las técnicas planteadas se tomó la metodología propuesta por Sapag Chaing (19).

Existen distintos procedimientos de cálculo según los diferentes tipos de costos e inversiones que deben ser considerados. La mayoría de las inversiones de un proyecto se concentran en aquellas que se deben realizar antes del inicio de la operación, aunque es importante considerar también las que se deben realizar durante la operación del proyecto, tanto por la necesidad de reemplazar activos como para enfrentar la ampliación proyectada del nivel de actividad.

Los costos de inversión incluyen los elementos e insumos necesarios para construir los drenes, las adecuaciones, modificaciones e instalación de los mismos. Todos estos recursos deben cuantificarse y valorarse a precios de mercado.

Además, se consideraron los costos anuales de operación y mantenimiento que contemplan todos los costos vinculados al funcionamiento del sistema de drenaje, así como también los materiales e insumos necesarios y remuneraciones al personal que debe operar los mismos.

Posteriormente, a partir del método de actualización de los valores, que consiste en calcular el valor actual de una eroga- ción o ingreso futuro, se aplicó el criterio del Valor Actual Neto (VAN) de las erogaciones de ambas técnicas para comparar y determinar la alternativa más conveniente, es decir la de menor VAN.

Para evaluar ambas alternativas se aplicó una tasa de corte del 10\% y 15 años como horizonte de planificación. Dado que las alternativas de drenaje son inversiones de diferente duración, se homologó la misma, de acuerdo a la metodología propuesta por Frank (9).

También se utilizó como un criterio de evaluación complementario, el método del Costo Operativo propuesto por Frank (9) que consiste en determinar el costo operativo anual de una inversión.

La anualidad incluye no solo el monto anual uniforme, pagadero en una determinada cantidad de años, que equivale a una inversión dada sino también, gastos anuales expresados en \$/año. Para ello se consideró la duración en años de cada técnica y una tasa del $10 \%$ obteniendo los costos operativos, expresados en pesos por año.

Para la estimación de los resultados se consideraron precios expresados en pesos correspondientes al mes de agosto de 2018.

\section{RESULTADOS Y DISCUSIÓN}

Para el diseño de DT se utilizó información planialtimétrica y de campo obtenida en investigaciones previas (3) resultando un espaciamiento entre drenes de $8 \mathrm{~m}$, profundidad de 0,6 m, con la opción de extremos finales abiertos (descarga libre) reforzados con tubos de PVC de $110 \mathrm{~mm}$. Los mismos se encuentran conectados a un canal colector o dren secundario excavado con profun- 
didades mínimas y máximas de 0,6 m y 0,9 $\mathrm{m}$ respectivamente, cuyo diseño hidráulico permitiría conducir tanto el escurrimiento superficial - lluvia con probabilidad de excedencia de 20\%-, como el excedente subsuperficial. Este canal se conecta a una cuneta de desagüe a través de un dispositivo que permite regular el flujo de salida -tubo con compuerta automática, la cual solo puede abrirse cuando el nivel de agua de la cuneta es menor que dentro del canal, de modo que se puede laminar el exceso de agua desde el lote hacia la red de drenaje exterior.

Para el diseño de DTu se utilizó la misma información de base que DT, adoptando 1,5 m como profundidad de instalación de los tubos, considerando a ésta como la mínima profundidad de la freática necesaria para lograr un desarrollo adecuado de pasturas en base a alfalfa en la región de estudio (1).

El espaciamiento y diámetro del tubo se obtuvieron a partir de la ecuación de Hooghoudt (3) y del área de aporte respectivamente. Para evitar el ingreso de material fino en el interior de los tubos se tuvo en cuenta una envoltura de geotextil seleccio- nada de acuerdo al diámetro efectivo de las partículas del suelo.

Los drenes parcelarios descargan a un dren secundario o colector abierto, de mayor capacidad que el construido para DT, que permite la conducción del excedente hídrico superficial (lluvia con probabilidad de excedencia de $20 \%$ ) y conectado a la red de drenaje exterior con similar dispositivo de tubo compuerta.

Un esquema con las técnicas evaluadas se presenta en la Figura 3.

Para la estructuración de los costos de inversión asociados a cada técnica bajo análisis se tomaron en cuenta las referencias que se detallan en la Tabla 1.

El costo del ítem A Estudios preliminares fue el mismo para ambas prácticas de drenaje, en tanto que los costos de los ítems B y C para DT fueron obtenidos según el diseño planteado (Figura 3b) y con precios de mercado (Tabla 2), utilizando para el caso del sistema DT un arado topo arrastrado por un tractor de 160 HP y retroexcavadora con pala frontal para construir el canal excavado y acarreo de suelo.
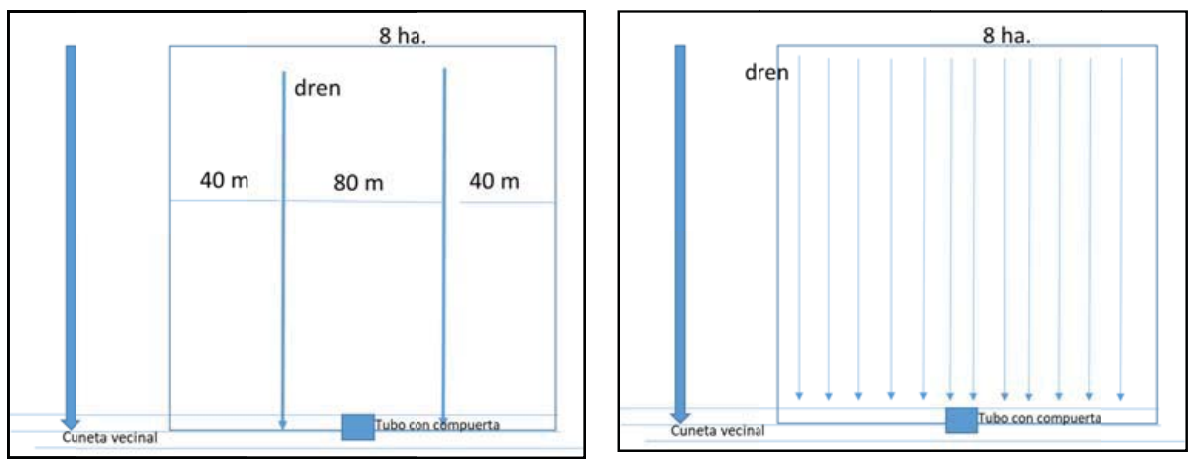

Figura 3: Esquema de diseño de Dren Tubería (DTu) a) y Dren Topo (DT) b).

Fuente: Elaboración propia. 


\section{G. Camussi et al.}

Tabla1: Variables consideradas para estimar los costos de inversión de las técnicas DT y DTu.

\begin{tabular}{|l|c|c|}
\hline \multicolumn{1}{|c|}{ Variables } & Dren topo & $\begin{array}{c}\text { Dren con } \\
\text { tuberías }\end{array}$ \\
\hline Unidad de análisis (ha) & 8 & 8 \\
\hline Espaciamiento entre drenes (m) & 8 & 80 \\
\hline Profundidad (m) & 0,6 & 1,5 \\
\hline Vida útil (años) & 3 & $15-20$ \\
\hline Mantenimiento & - & anual \\
\hline
\end{tabular}

Fuente: Elaboración propia.

Tabla 2: Estructura de costo de inversión en dren topo (DT)

\begin{tabular}{|c|c|c|}
\hline Items & Unidad & Costo total \\
\hline \multicolumn{3}{|l|}{ A) Estudios preliminares } \\
\hline Estudios planimetrícos & $\$$ & 1.807 \\
\hline Estudios de campo & $\$$ & 2.000 \\
\hline \multicolumn{3}{|l|}{ B) Obra de drenaje } \\
\hline $\begin{array}{l}\text { Diseño de red de drenaje } \\
\text { Replanteo de red de drenaje a campo } \\
\text { Realización de labor }\end{array}$ & $\$$ & 4.072 \\
\hline \multicolumn{3}{|c|}{ C) Obra: Cons trucción de zanja excavada } \\
\hline $\begin{array}{l}\text { Materiales: } \\
\text { Tubo compuerta }\end{array}$ & $\$$ & 4.356 \\
\hline Tubo de $110 \mathrm{~mm}$ & $\$$ & 6.909 \\
\hline Costo de excavación & $\$$ & 52.000 \\
\hline Control de obra & $\$$ & 3.000 \\
\hline Total de costo de inversión & $\$$ & 74.144 \\
\hline Total de costo de inversión & \$/ha & 9.268 \\
\hline
\end{tabular}

Fuente: Elaboración propia. 
Para DTu el costo de labor del ítem B, Obra de drenaje, es sustancialmente mayor que DT porque se requiere mayor cantidad de horas de trabajo para realizar la zanja donde se colocan los tubos que luego tiene que ser rellenada. También se consideró la mano de obra para envolver el geotextil. El costo de los materiales incluye tubo corrugado de polietileno de alta densidad de $100 \mathrm{~mm}$ de diámetro, acoples y geotextil (Tabla 3).
La excavación del dren secundario representa el 85\% de los costos en DT (Figura 2 A) con lo cual, una pequeña variación en su precio afecta considerablemente el valor final de la inversión. Otras alternativas que implicarían menores costos de inversión p.e. no construir zanja colectora al final de los DT, o bien construir zanjas menos profundas están siendo evaluadas en condiciones reales de campo.

Tabla 3: Estructura de costos de inversión en dren con tubería (DTu).

\begin{tabular}{|c|c|c|c|}
\hline Items & Unidad & $\begin{array}{c}\text { Costo unitario } \\
\$ / \text { unidad }\end{array}$ & $\begin{array}{c}\text { Costo total } \\
\$\end{array}$ \\
\hline \multicolumn{4}{|l|}{ A) Estudios preliminares } \\
\hline Estudios planimetrícos & $\$ /$ ha & 286 & 1.807 \\
\hline Estudios de campo & \$/ha & 250 & 2.000 \\
\hline \multicolumn{4}{|l|}{ B) Obra de drenaje } \\
\hline $\begin{array}{l}\text { Diseño de red de drenaje } \\
\text { Replanteo de red de drenaje a campo }\end{array}$ & $\$$ /ha & 282 & 2.258 \\
\hline \multicolumn{4}{|l|}{ Costo de labor } \\
\hline Alquiler de servicio de excavado & $\$ / h$ & 1.500 & 60.000 \\
\hline Colocación de geotextil y tubos & $\$ / d$ & 700 & 700 \\
\hline \multicolumn{4}{|l|}{ Materiales } \\
\hline Tuberías & $\$ / 50 \mathrm{~m}$ & 7.034 & 140.681 \\
\hline Acoples & $\$$ & 66 & 1.316 \\
\hline Geotextil & $\$ / 10 \mathrm{~m}$ & 602 & 60.200 \\
\hline \multicolumn{4}{|c|}{ C) Obra: Construcción de zanja excavada } \\
\hline Costo de excavación & $\$ / m^{3}$-ha & 155 & 103.999 \\
\hline Tubo compuerta & \$/unidad & 5.750 & 5.750 \\
\hline Control de obra & $\$ / d$ & 6.000 & 3.000 \\
\hline \multicolumn{4}{|l|}{ D) Costos de mantenimiento } \\
\hline Mantenimiento anual & $\$ / d$ & 6.000 & 6.000 \\
\hline Total Costo de inversión & $\$$ & & 387.711 \\
\hline Total Costo de inversión & \$/ha & & 48.464 \\
\hline
\end{tabular}

Fuente: Elaboración propia. 


\section{G. Camussi et al.}

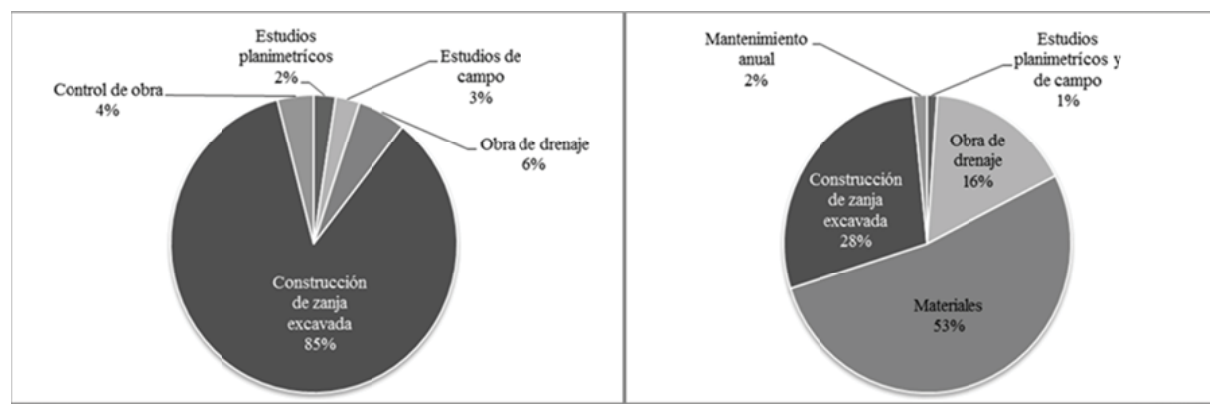

Figura 4: Incidencia de cada rubro sobre el costo total de la inversión (en \%).

Fuente: Elaboración propia.

Por el contrario en DTu el costo del dren o colector secundario representa solo $28 \%$, teniendo la mayor incidencia sobre el costo total de la inversión los materiales necesarios para la obra, los que constituyen más del $50 \%$ en el caso analizado.

El costo de inversión para DTu en este trabajo resultó mayor comparado con un sistema de drenaje subsuperficial similar que está instalado y en funcionamiento en un establecimiento azucarero de Jujuy (21), 1.200 U\$S vs 900 U\$S ha-1 respectivamente. La diferencia encontrada responde parcialmente a que la empresa de referencia ha invertido en maquinaria específica y en la fabricación de parte de los materiales (tubos de diferente diámetro) lo cual disminuiría los costos.

Los costos de sistemas de drenaje están compuestos por factores variables tales como el tipo de suelo, accesibilidad al sitio, espaciamiento o densidad de drenes, profundidad, diámetro de los mismos, tipo de material filtrante , extensión del o de los drenes secundarios o colectores (canales abiertos), disponibilidad de insumos y experiencia en los trabajos de drenaje (8). A su vez, la calidad de los materiales usados también anterior. afectan los costos según el sistema aplicado. Por ende, hay mucha variabilidad y refieren siempre a una situación particular, y en este caso, al predio estudiado.

Crosson P (6) et al. señalan, para el Sistema de DT, costos 38\% inferiores de los aquí calculados. Sin embargo, no pueden compararse estrictamente dado lo mencionado anteriormente, como por no ser similares las características técnicas del sistema propuesto.

Para evaluar las alternativas se aplicó en primer término el método de actualización de los valores (VAN). Los resultados obtenidos, que se detallan en la Tabla $\mathrm{N}^{\circ} 4$, muestran que el VAN de la técnica correspondiente a DT es un 25\% menor (VAN: \$106.319) que en el caso de DTu (VAN: \$433.348) por lo que resultaría aquel la mejor alternativa desde el punto de vista de la inversión y de los costos asociados.

Posteriormente se evalúo la inversión considerando el método del Costo Operativo cuyos resultados se presentan en la tabla No5 donde queda expuesto que el Costo Operativo de la Técnica DTu es un 50\% superior a la técnica DT como se detecta en el análisis 
Tabla 4: Valor de la inversión y VAN

\begin{tabular}{|l|r|r|r|}
\hline \multicolumn{1}{|c|}{ Proyecto } & Inversión (\$) & VAN (\$) & VAN (\$/ha) \\
\hline Dren topo & 74.144 & 106.319 & 13.290 \\
\hline Dren entubado & 387.711 & 433.348 & 54.168 \\
\hline
\end{tabular}

Fuente: Elaboración propia.

Tabla 5: Costo Operativo de la inversión

\begin{tabular}{|l|c|c|c|}
\hline \multicolumn{1}{|c|}{ Proyecto } & $\begin{array}{c}\text { Inversión } \\
\text { (\$) }\end{array}$ & $\begin{array}{c}\text { Costo Operativo } \\
\text { (S/año) }\end{array}$ & $\begin{array}{c}\text { Costo Operativo } \\
\text { (\$/ha-año) }\end{array}$ \\
\hline Dren topo & 74.144 & 29.814 & 3.727 \\
\hline Dren entubado & 381.711 & 56.183 & 7.023 \\
\hline
\end{tabular}

Fuente: Elaboración propia.

Considerando los resultados obtenidos, ambas metodologías, VAN y Costo operativo, arrojan menores costos en DT. En la preferencia por una u otra técnica, deberán tenerse en cuenta la posibilidad y costo de financiamiento, complejidad administrativa, disponibilidad de recursos humanos capacitados, entre otros factores propios de cada sistema productivo.

Además de las técnicas evaluadas existen otras alternativas para mitigar los efectos económicos de los excesos hidricos. Entre las cuales se puede mencionar los seguros multiriesgo que cubren riesgos, climáticos (granizo, incendio, inundación, sequía, vientos, heladas, falta de piso y lluvias en exceso) y/o biológicos. Los mismos cubren la inversión del productor para gastos de implantación y produccion del cultivo (16).

\section{CONCLUSIONES}

Los costos de operación y montos de inversión son significativamente diferentes entre DT y DTu, requiriendo fuentes de financiamiento adecuadas para su implementación, especialmente para el caso de DTu.

Es importante destacar que sin la implementación de alguna de estas técnicas el productor queda expuesto a pérdidas productivas y económicas que pueden condicionar la viabilidad de la empresa.

Resulta necesario relevar información en condiciones reales sobre el impacto de ambas técnicas en relación a la productividad de las pasturas y producción de carne o leche para realizar una evaluación económica que permita su comparación con otras alternativas de mitigación de excesos hídricos. 
Si bien las técnicas DT y DTu mitigan problemas de encharcameinto y anegamiento a nivel predial, su implementación debe adecuarse a las normativas vigentes.

\section{BIBLIOGRAFÍA}

1.- BERHONGARAY, G.; BASANTA, M.; JOSÉ MARTÍN JAUREGUI, J. 2019. Water table depth affects persistence and productivity of alfalfa in Central Argentina. Journal homepage: www.elsevier.com/locate/fcr. Acceso: 24/2/19.

2.- CAMUSSI G. Y R. MARANO. 2008. Respuesta del trigo al riego suplementario en la región central de Santa Fe. Revista FAVE Sección Ciencias Agrarias Volumen 7 (1y2): 7-21.

3.- CAMUSSI, G. F.; MARANO, R.P., GHIBERTO, P. 2018 Drenaje subsuperficial en un argiudol ácuico de la región central de Santa Fe. Congreso Argentino de la Ciencia del Suelo. Tucumán, 15 al 18 de mayo de 2018 ISBN 978-987-46870-0-5

4.- CAVELAARS, J.C.; VLOTMAN, W.F. Y SPOOR, G. 2006. Subsurface Drainage Systems. En: H.P.Ritzema (Ed.), Drainage Principles and Applications. International Institute for Land Reclamation and Improvement (ILRI), Publication 16, third revised edition, p. 827-928. Wageningen, The Netherlands. ISBN 9070754339.

5.- CHOW, VEN TE; MAIDMENT, D.R.; MAYS, L.W. 1994. Hidrología Aplicada. ISBN: 958-600-171-7. Trducción: Saldarriaga, J. Colombia. 577 pp.

6.- CROSSON, P., SHALLOO, L., THORNE, F. 2013. The economics of land drainage. In: Moore,M., Fenton, O., Tuohy, P., Ibrahim, T. (Eds.), Teagasc Manual on Drainage and soil management: A best practice manual for Ireland's Farmera. Teagasc,Carlow, Ireland, pp. 113-119.
7.- FERTONANI M.E. \& H.H. PRENDES. 1983. Hidrología en áreas de llanura, aspectos conceptuales teóricos y metodológicos. En: Hidrología de las grandes llanuras (Ed. M.C. Fuschini Mejía). Actas del Coloquio de Olavarría. Vol.I. Olavarría, Argentina. pp. 119-155.

8.- FITZGERALD, J. B., BRERETON, N., HOLDEN, M. 2008. Simulation of the influence of por soil drainage on grass-based dairy production systems in Irland. Grass Forrage Sci. 63,380-389. Disponible en: https:// doi.org/10.1111/j.1365-2494.2008.00637.x. Acceso: 26/2/19.

9.- FRANK, R. 1998. Evaluación de inversiones en la empresa agraria. El Ateneo. Buenos Aires. $104 \mathrm{p}$.

10.- FUSCHINI MEJÍA, M. 1994. El agua en las llanuras. UNESCO - Programa Hidrológico Internacional. 58 p. Montevideo.

11.- GASTALDI, L. B. 2017. Lechería pampeana. Resultados del ejercicio 2016- 2017. Informe Técnico. (online). Disponible en: https:// inta.gob.ar/sites/default/files/inta_encuesta_lechera_2016_2017_informe_tecnico_version_20_abr_18.pdf. Acceso: 24/2/19.

12.- LOVINO, M. A. 2015. Impacto ambiental de la variabilidad climática y los eventos extremos en la provincia de Santa Fe, en el contexto del cambio climático. Tesis Doctoral, UNL, Argentina, 238 pp. http://bibliotecavirtual.unl. edu.ar:8080/tesis/handle/11185/707

13.- MAGRIN, G.O. et. al. 2014. Centro y Sudamérica. En: Barros, VR, et al., Eds., Cambio Climático 2014: Impactos, Adaptación y Vulnerabilidad, Parte B: Aspectos Regionales, Cambridge University Press, Cambridge, 1499-1566

14.- MARANO R. \& G. CAMUSSI. 2017 Técnicas integradas de manejo del agua predial para sistemas agrícolas y ganaderos. Publicaciones Miscelaneas. 
15.- MARANO, R.P. 1996. Riego suplementario en la cuenca lechera del centro-este Santa Fe.” Revista Miscelánea No 77. EEA INTA Rafaela.

16.- MIGUEZ, D. 2017. Jornada de riesgo agropecuario. Los Seguros Agropecuarios en la Argentina. Mercado, Situación Actual y Tendencias. En: http://www.ora.gob.ar/seguros_ general.php.

17.- MONTICO, S. 2004. El manejo del agua en el sector rural de la región Pampena argentina. Revista THEOMAI.

18.- MOORE,M.; FENTON, O.; TUOHY, P., \& IBRAHIM, T. 2013. Teagasc Manual on Drainage and soil management: A best practice manual for Ireland's Farmera. Teagasc, Carlow, Ireland, $44 \mathrm{pp}$

19.- NASSIR SAPAG CHAIN. 2011. Proyectos de inversión. Formulación y evaluación. Pearson Educación. Chile. 544 p.

20.- OOSTERBAAN, R. 1991. Agricultural Land Drainage: a wider application through caution and restraint. ILRI Annual Report 1991, p.21-36, International Institute for Land Reclamation and Improvement, Wageningen, The Netherlands.
21.- RECUPERACIÓN Y CONSERVACIÓN DE SUELOS MEDIANTE EL DRENAJE SUBTERRÁNEO - TEMÁTICA LEDESMA S.A.A.I. 2004. Recuperación y Conservación de Suelos mediante el Drenaje Subterráneo - Temática "Casos de Ecoeficiencia”. (online) Disponible en: http://produccion.jujuy. gob.ar/wp-content/uploads/sites/18/2016/09/ Ledesma.Ecoeficiencia.pdf. Acceso: 24/2/19.

22.- TUOHY, P., HUMPHREYS, J., HOLDEN, N. M., FENTON, O. 2016. Runoff and subsurface drain response from mole and gravel mole drainage across episodic rainfall events. Agric. Water Manag. 169, 129-139.

23.- YIN, R. K.1994. Case Study Research: Design and Methods. Sage Publications, Thousand Oaks, CA. 